\title{
INTEGRAÇÃO NUMÉRICA DE LEIS DE VELOCIDADE DIFERENCIAIS COM O USO DO SCILAB
}

Rafael Donizete Dutra Sandes e Renato Canha Ambrosio*

Departamento de Química, Universidade Federal de Sergipe, Cidade Universitária Prof. José Aloísio de Campos, Av. Mal. Rondon, s/n, 49100-000 São Cristóvão - SE, Brasil

Camilo Andrea Angelucci

Centro de Ciências Naturais e Humanas, Universidade Federal do ABC, Rua Santa Adélia, 166, 09210-170 Santo André - SP, Brasil

Recebido em 11/5/12; aceito em 23/8/12; publicado na web em 28/11/12

\begin{abstract}
NUMERICAL INTEGRATION OF DIFFERENTIAL RATE LAWS BY MEANS OF SCILAB. In this work, we applied the free open source SCILAB software for the numerical integration of differential rate law equations to obtain the concentration profiles of chemical species involved in the kinetics of some complex reactions. An automated method was applied to construct the system of ordinary differential equations (ODE) from the postulated chemical models. The solutions of the ODEs were obtained numerically by standard SCILAB functions. We successfully simulated even complex chemical systems such as $\mathrm{pH}$ oscillators. This communication opens up the possibility of using SCILAB in simulations and modeling by our chemistry undergraduate students.
\end{abstract}

Keywords: oscillating reactions; numerical integration; SCILAB.

\section{INTRODUÇÃO}

A cinética química está relacionada ao estudo do comportamento das variações das concentrações das espécies químicas envolvidas em uma reação com respeito ao tempo. ${ }^{1-8} \mathrm{~A}$ velocidade de consumo ou produção das espécies é expressa a partir de equações diferenciais ordinárias (EDO) denominadas de leis de velocidade diferenciais (LDV). ${ }^{2}$ A obtenção dos perfis de concentração em função do tempo para as espécies químicas envolvidas na reação é realizada a partir da integração das leis de velocidade diferenciais, resultando nas leis de velocidade integradas (LVI), funções matemáticas que relacionam as concentrações das espécies com o tempo. ${ }^{1,2}$

É interessante destacar que os livros de físico-química empregados nos cursos de graduação em Química seguem, de modo geral, sempre o mesmo enfoque: aplicar as LVI linearizadas aos resultados experimentais de concentração em função do tempo. Se foi obtido um ajuste desses dados às LVI, prossegue-se em determinar a ordem global da reação e a constante de velocidade..$^{1-8}$

Apesar de o grau da equação diferencial que compõe a LVD ser determinado pelo mecanismo da reação, as concentrações dos intermediários que o compõe não devem aparecer na lei de velocidade, tanto em sua forma diferencial, quanto na forma integrada. ${ }^{1,2}$ Talvez por este motivo a abordagem feita pela maioria dos livros de graduação seja bastante simplista, negligenciando os perfis de concentração de intermediários, isto é, como variam as concentrações destes com o tempo. Este lapso de informação fica patente quando se introduz o conceito de mecanismo reacional. É fácil observar que, para nossos alunos de graduação, as espécies intermediárias são apenas compostos produzidos entre reagentes e produtos, sendo de pouca importância, esquecendo-se que fenômenos extremamente interessantes, como o das reações oscilantes, resultam de oscilações nas concentrações de intermediários no decorrer da reação. ${ }^{2,9,10}$

Para obtermos o perfil de concentração de uma espécie envolvida em um mecanismo de reação, devemos expressar sua velocidade de produção/consumo com as concentrações das espécies que participam das etapas elementares que resultem em sua produção ou consumo. Vamos considerar um mecanismo simples composto de duas reações

*e-mail: renatocanha@gmail.com elementares e consecutivas de primeira ordem:

$$
\mathrm{A} \stackrel{\mathrm{k} 1}{\rightarrow} \mathrm{B} \stackrel{\mathrm{k} 2}{\rightarrow} \mathrm{C}
$$

As velocidades de consumo de A, formação e consumo do intermediário B e geração de C são dadas pelo sistema de EDO's:

$$
\begin{gathered}
\frac{d[A]}{d t}=-k_{1}[A] \\
\frac{d[B]}{d t}=k_{1}[A]-k_{2}[B] \\
\frac{d[C]}{d t}=k_{2}[B]
\end{gathered}
$$

A integral da primeira equação diferencial do sistema é bem conhecida:

$$
[A]=[A]_{0} e^{-k_{1} t}
$$

e, quando substituída na segunda equação diferencial resulta:

$$
\frac{d[B]}{d t}+k_{2}[B]=k_{1}[A]_{0} e^{-k_{1} t}
$$

A solução analítica da Equação 5 é muito bem descrita na literatura. ${ }^{11} \mathrm{~A}$ função que rege a concentração de $\mathrm{B}$ em função do tempo resulta em:

$$
[B]=[B]_{0} e^{-k_{2} t}+\frac{k_{1}[A]_{0}}{k_{2}-k_{1}}\left(e^{-k_{1} t}-e^{-k_{2} t}\right)
$$

A concentração do produto $\mathrm{C}$ pode ser encontrada pelo balanço de concentrações, isto é, $[C]=[A]_{0}+[B]_{0}+[C]_{0}-[A]-[B]$, o que nos leva a:

$[C]=[A]_{0}\left(1-\frac{1}{k_{2}-k_{1}}\left(k_{2} e^{-k_{1} t}-k_{1} e^{-k_{2} t}\right)\right)+[B]_{0} e^{-k_{2} t}+[C]_{0}$ 
No entanto, a maioria dos mecanismos reacionais é formada por inúmeras etapas elementares, muitas vezes de molecularidade superior a 1, resultando na necessidade de inúmeras EDO's não lineares para descrever a evolução temporal da concentração de cada espécie envolvida no processo. ${ }^{9,10,12} \mathrm{O}$ problema que advém desses casos é que a integração do sistema de equações diferenciais, que define as variações temporais das concentrações, se torna impossível de ser efetuada por métodos de cálculo elementar, tornando necessário o emprego de métodos numéricos para a obtenção dos perfis de concentração. ${ }^{12-14}$ Neste ponto, é importante frisar que a literatura empregada no ensino de cinética química negligencia este tipo de problema. Podemos apontar duas prováveis razões para tal omissão: o desconhecimento dos fundamentos da integração numérica pela maioria dos alunos dos cursos de graduação em Química e a falta de noções mínimas de linguagem computacional no uso de softwares de cálculo numérico. Alguns trabalhos na literatura evidenciam também essa falha presente no ensino de química e, de modo geral, utilizam de metodologias computacionais para auxiliar no ensino e aprendizagem desses problemas..$^{15-18}$

Diante da perspectiva apresentada, desenvolvemos este trabalho com o intuito de apresentar exemplos de mecanismos de reações complexas, para os quais não há uma solução analítica para as equações diferenciais e, ao mesmo tempo, introduzir um método geral para a resolução desse tipo de problema através da plataforma de programação SCILAB. ${ }^{19}$

Destacaremos casos pouco abordados nos cursos de graduação, como mecanismos envolvendo reações competitivas de segunda ordem e, com maior ênfase, reações oscilantes em sistemas fechados e em reatores agitados de fluxo contínuo (RAFC). Desta forma, esperamos contribuir para a melhoria na qualidade dos cursos de cinética química em nível de graduação e a difusão do uso da plataforma de programação livre SCILAB em nossas Universidades.

\section{FERRAMENTAS COMPUTACIONAIS}

\section{Integração numérica}

De modo a deixar o assunto mais palatável ao leitor, é interessante que este tenha algum conhecimento de cálculo numérico, no entanto, essa falta não implica em prejuízo no entendimento deste artigo. Resumidamente, o conceito de integração numérica pode ser entendido através do método de Euler, que usa uma forma da expansão em séries de Taylor, truncada na primeira derivada. ${ }^{2,12,13,20}$ Se a concentração de um reagente $\mathrm{A}$ for uma função da variável tempo, $[\mathrm{A}](\mathrm{t})$, então a concentração no tempo $\mathrm{t}+\Delta \mathrm{t},[\mathrm{A}](\mathrm{t}+\Delta \mathrm{t})$, pode ser estimada pela derivada da concentração no tempo t, $(d[A] t) / d t)$ :

$$
[A](t+\Delta t) \approx[A](t)+\left(\frac{d[A](t)}{d t}\right) \Delta t
$$

Em outras palavras, o método consiste em encontrar aproximações para $[\mathrm{A}](\mathrm{t}+\Delta \mathrm{t})$ a partir da extrapolação linear $[\mathrm{A}](\mathrm{t})$ ao longo da linha definida pela sua tangente no tempo $t$, conforme ilustra a Figura 1. O princípio é aplicado em várias etapas (iterações), desde o tempo inicial até o tempo final, e em cada iteração o valor estimado de $[\mathrm{A}](\mathrm{t}+\Delta \mathrm{t})$ na iteração anterior é usado para estimar $[\mathrm{A}](\mathrm{t}+2 \Delta \mathrm{t})$ e assim por diante. ${ }^{20}$ Assim, o método de Euler permite calcular a concentração de A ao longo do tempo. Os métodos de integração numérica modernos empregam outras técnicas, que incluem estratégias como a utilização dos termos de ordem superior da expansão em séries de Taylor ou o cálculo da primeira derivada em múltiplos pontos dentro de um intervalo de tempo. . $^{12,13,20}$

Para melhor ilustrar o método numérico como ferramenta na

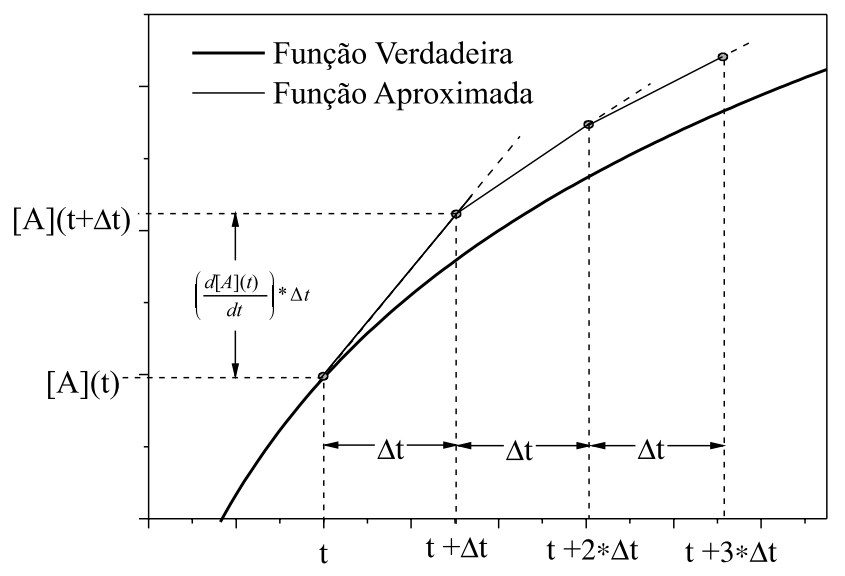

Figura 1. Ilustração de integração numérica baseado no método de Euler

obtenção dos perfis temporais das espécies envolvidas num mecanismo reacional, vamos usar o exemplo apresentado na Introdução para reações consecutivas de primeira ordem (reação 1). Na Figura 2 estão representadas as soluções analíticas previamente apresentadas (Equações 4, 6 e 7) bem como o resultado da integração numérica para este exemplo. Note que a simulação resulta em um perfil de consumo/produção das espécies em função do tempo que se ajusta perfeitamente às soluções analíticas.

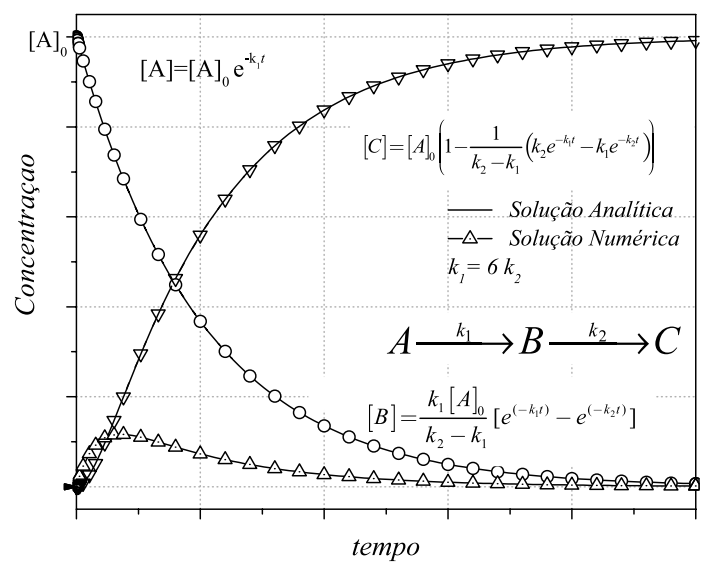

Figura 2. Perfis de concentração pelo tempo para uma reação consecutiva de primeira ordem $(A \rightarrow B \rightarrow C)$. Comparação entre solução analítica e solução pelo método numérico

\section{O SCILAB como ferramenta no ensino de cinética química}

Utilizamos a poderosa ferramenta computacional gratuita SCILAB por ser um ambiente de programação interativo de alto desempenho voltado para o cálculo numérico, cujas entradas de dados são sempre feitas na forma matricial. O SCILAB dispõe de uma extensa biblioteca de funções para resolução de diversos problemas numéricos, além de vários módulos especializados para finalidades diversas como, por exemplo, processamento de sinais e de imagens e criação de interface gráfica ao usuário. A linguagem empregada na programação SCILAB é relativamente simples, facilmente compreendida por alunos de graduação.

Do ponto de vista computacional, o primeiro passo na integração numérica é construir o sistema de equações diferenciais ordinárias que representa o mecanismo ou o modelo da reação. Se utilizarmos softwares como Mathematica ${ }^{\circledR}$ ou Mathcad ${ }^{\circledR}$, o procedimento é manual e sujeito a equívocos, especialmente para mecanismos de reações complexas envolvendo várias espécies químicas e várias etapas 
elementares. ${ }^{18}$ Para facilitar o entendimento do leitor e tornar o método de integração mais acessível, empregamos uma versão modificada do algoritmo matricial proposto originalmente por Puxty et al., ${ }^{12}$ em que as equações diferencias são construídas automaticamente a partir dos coeficientes estequiométricos das etapas elementares que compõem o mecanismo de reação. Vale ressaltar que o software criado possui uma interface gráfica bastante amigável e o SCILAB ${ }^{19}$ é apenas a "plataforma" para executar nosso software, não exigindo conhecimento de linguagem de programação por parte do usuário. Os fundamentos do método empregado serão apresentados na próxima seção e o software é distribuído livremente pelos autores deste artigo.

\section{EXEMPLOS DE APLICAÇÕES}

\section{Reações consecutivas, competitivas de segunda ordem}

As leis de velocidade do mecanismo de reação apresentado na Introdução são equações diferenciais lineares em que suas integrais podem ser escritas na forma analítica e, portanto, o uso do computador não oferece vantagem significativa, exceto pelo fato de facilitar a visualização dos perfis de concentração. A complexidade aumenta na medida em que o mecanismo apresenta etapas elementares de molecularidades mais elevadas, a exemplo das reações consecutivas e competitivas de segunda ordem:

$$
\begin{aligned}
& A+B \stackrel{k_{1}}{\longrightarrow} C \\
& A+C \stackrel{k_{2}}{\longrightarrow} D
\end{aligned}
$$

Esse mecanismo se aplica a reações de saponificação de diésteres, onde A representa o íon hidróxido, $\mathrm{B}$ o diéster, $\mathrm{C}$ o monoéster e D o ácido dicarboxílico. ${ }^{21} \mathrm{O}$ sistema de EDO's que resulta do mecanismo formado pelas reações elementares reações 2 e 3 não possuem solução analítica. Dessa forma, utilizaremos esse mecanismo para apresentar a entrada de dados para a resolução pelo método numérico. A metodologia empregada para esse exemplo será utilizada em outros mecanismos de maior complexidade discutidos nas seções seguintes.

$\mathrm{O}$ método prático de declaração das equações diferenciais começa com a construção de duas matrizes, $X_{R}$ e $X_{p}$, que armazenam os coeficientes estequiométricos dos reagentes e produtos, respectivamente, das etapas elementares reações 2 e 3 que compõem o mecanismo. ${ }^{12} \mathrm{O}$ elemento na posição $(j, i)$ da matriz $\mathrm{X}_{\mathrm{R}}$ corresponde ao coeficiente estequiométrico $\left(x r_{j i}\right)$ da $i$-ésima espécie química, que participa como reagente na $j$-ésima etapa elementar do mecanismo; caso não participe como reagente, a posição correspondente é preenchida com zero. No exemplo em questão temos duas etapas (reações 1 e 2) com 4 espécies químicas no total: A, B, C e D. Os elementos presentes na $1^{\mathrm{a}}$ e a $2^{\mathrm{a}}$ linhas da matriz $\mathrm{X}_{\mathrm{R}}$ são representados pelos coeficientes estequiométricos $\left(x r_{j i}\right)$ de cada espécie presente como reagentes nas reações 1 e 2, respectivamente. Para a espécie química A, que participa como reagente da etapa (reação 1) do mecanismo, o coeficiente estequiométrico é $1, x r_{l A}=1$, para B $x r_{l B}=1$; ao passo que para C e D os valores são nulos $\left(x r_{j C}=0\right.$ e $\left.x r_{j D}=0\right)$, resultando na matriz $\mathrm{X}_{\mathrm{R}}$ :

$$
X_{R}=\left[\begin{array}{cccc}
x r_{1 A} & x r_{1 B} & x r_{1 C} & x r_{1 D} \\
1 & 1 & 0 & 0 \\
x r_{2 A} & x r_{2 B} & x r_{2 C} & x r_{2 D} \\
1 & 0 & 1 & 0
\end{array}\right](r .1)
$$

Da mesma forma se procede na construção da matriz $\mathrm{X}_{\mathrm{p}}$, onde os coeficientes estequiométricos das respectivas espécies como produtos nas etapas elementares correspondentes ocupam as posições $\left(x p_{j i}\right)$ :

$$
X_{P}=\left[\begin{array}{cccc}
x p_{1 A} & x p_{1 B} & x p_{1 C} & x p_{1 D} \\
0 & 0 & 1 & 0 \\
x p_{2 A} & x p_{2 B} & x p_{2 C} & x p_{2 D} \\
0 & 0 & 0 & 1
\end{array}\right](r .1)
$$

Resumidamente, o número de linhas das matrizes é igual ao número de etapas elementares $\left(n_{r}\right)$ ao passo que o número de colunas equivale ao número de espécíes químicas $\left(n_{e}\right)$ que tomam parte no mecanismo ou no modelo da reação.

Uma vez que as matrizes $X_{R}$ e $X_{\mathrm{P}}$ são declaradas pelo usuário no próprio programa, $\mathrm{X}_{\mathrm{R}}$ é subtraída de $\mathrm{X}_{\mathrm{P}}$ resultando na matriz $\mathrm{X}$ :

$$
X=\left[\begin{array}{llll}
-1 & -1 & 1 & 0 \\
-1 & 0 & -1 & 1
\end{array}\right]
$$

A matriz $X$, resultado da operação algébrica de subtração de $X_{R}$ de $X_{P}$, é utilizada para construção das equações diferenciais que representam as velocidades de consumo e produção de cada espécie e as velocidades de reações das etapas presentes no mecanismo reacional. De maneira generalizada, a construção de cada equação é feita através das Equações 9 e 10:

$$
\begin{aligned}
& v_{j}=k_{j} \prod_{i=1}^{n e}[u]_{i}^{x r_{j i}} \\
& \frac{d[u]}{d t}=\sum_{j=1}^{n r} X_{(j, i)} v_{j}
\end{aligned}
$$

onde $v_{j}$ e $k_{j}$ representam a velocidade e a constante de velocidade da $j$-ésima etapa do mecanismo, respectivamente; e $[u]$ a concentração da $i$-ésima espécie química. ${ }^{12}$

O índice $i$ presente nas Equações 9 e 10 indica a espécie química que participa como reagente da $j$-ésima etapa do mecanismo, isto é, $i=1$ para A, $i=2$ para B e assim por diante. Fazendo as devidas substituições, chegamos às leis de velocidade para cada etapa do mecanismo:

$$
\begin{aligned}
& v_{1}=k_{1}[A]^{1}[B]^{1}[C]^{0}[D]^{0} \\
& v_{2}=k_{2}[A]^{1}[B]^{0}[C]^{1}[D]^{0}
\end{aligned}
$$

Para obtermos as equações diferenciais que representam a variação temporal de cada espécie envolvida no mecanismo, substituímos as Equações 11 e 12 na Equação 10, resultando no sistema de equações diferenciais ordinárias:

$$
\begin{gathered}
\frac{d[A]}{d t}=(-1) v_{1}+(-1) v_{2} \\
\frac{d[B]}{d t}=(-1) v_{1}+(0) v_{2} \\
\frac{d[C]}{d t}=(1) v_{1}+(-1) v_{2} \\
\frac{d[D]}{d t}=(0) v_{1}+(1) v_{2}
\end{gathered}
$$

É importante ressaltar que o processo de montagem das equações diferenciais é automático no software que criamos e que é necessário apenas declarar as matrizes dos coeficientes estequiométricos, além dos valores das constantes de velocidade nas janelas gráficas apropriadas. Uma vez montado o sistema e de posse de informações sobre as condições iniciais (concentrações dos componentes no tempo $t=0$ ), 
o software resolve o sistema usando a função "ode". A solução para o conjunto de EDO's é o perfil temporal de concentração de cada componente, e os resultados são apresentados na Figura 3. Neste ponto, o próprio software gera um gráfico com a evolução temporal de cada espécie; mas também há a possibilidade de salvar os dados gerados para serem utilizados em qualquer programa comercial para visualização e tratamento de dados. (Maiores detalhes quanto ao uso do software constam no material suplementar).

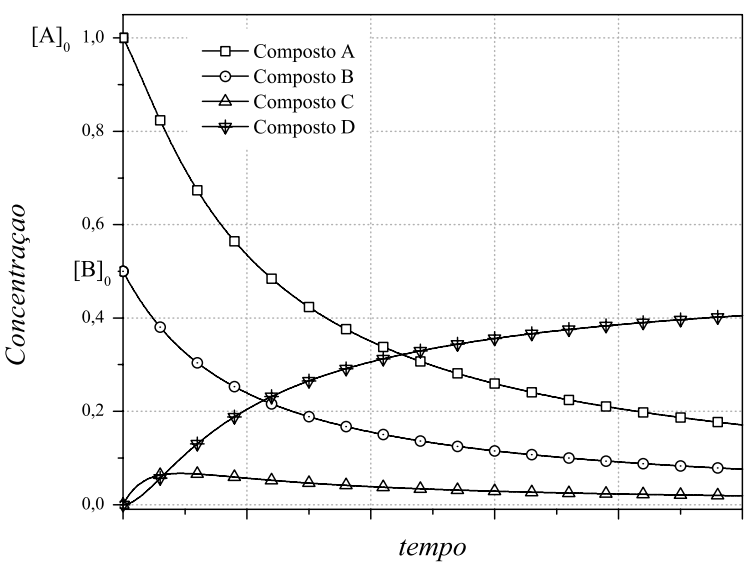

Figura 3. Perfis de concentração pelo tempo para uma reação consecutiva de segunda ordem. $\left.[A]_{0}=1 \mathrm{~mol} \mathrm{~L} L^{-1} ;[B]_{0}=0,5 \mathrm{~mol} \mathrm{~L} L^{-1} ; C\right]_{0}=0 \mathrm{~mol} \mathrm{~L}^{-1}$; $[D]_{0}=0 \mathrm{~mol} \mathrm{~L}^{-1} ; k_{2}=5 \cdot k_{1}$

Uma das vantagens do uso desse software está em explorar, por exemplo, o comportamento de cada espécie quando se variam as constantes de velocidade envolvidas bem como as concentrações iniciais de cada componente do mecanismo.

\section{Reações em reatores de fluxo contínuo agitado (RFCA)}

O exemplo que apresentamos acima diz respeito a uma reação conduzida em sistema fechado que, inexoravelmente, atinge o estado de equilíbrio químico. ${ }^{910}$ Para observar comportamentos mais interessantes, devemos simular sistemas abertos, isto é, sistemas que podem realizar trocas de matéria e energia com suas vizinhanças, sendo, portanto, mantidos afastados do equilíbrio termodinâmico. Sistemas reacionais podem ser mantidos afastados do estado de equilíbrio através, por exemplo, de uma alimentação constante de soluções reagentes através de bombas peristálticas. ${ }^{9,10,22}$ Esses sistemas são conhecidos como reatores de fluxo contínuo agitado (RFCA) e uma agitação eficiente é fundamental para evitar a formação de gradientes espaciais. ${ }^{9}$

Um parâmetro fundamental dos reatores que operam em fluxo contínuo é a constante de fluxo, $\mathrm{k}_{0}$, tomada como o quociente do fluxo de entrada de reagentes, F, e o volume do reator, $\mathrm{V}:{ }^{9,10,22,23}$

$$
k_{0}\left(s^{-1}\right)=\frac{F\left(m L \cdot s^{-1}\right)}{V(m L)}
$$

$\mathrm{O}$ recíproco de $\mathrm{k}_{0}$ é o tempo médio em que uma molécula passa no reator e um fluxo alto em um reator de grandes dimensões é equivalente, em termos dinâmicos, a um fluxo baixo em um reator de pequenas dimensões. ${ }^{9}$ Devido ao fluxo contínuo de reagentes, as moléculas passam apenas um tempo finito no reator. Para manter o volume do reator constante, é óbvio que a solução reacional deve escoar continuamente, removendo não apenas produtos, mas também reagentes não consumidos.

Vamos aplicar esse raciocínio a um mecanismo de reação constituído de uma etapa de autocatálise cúbica representando um processo de retroalimentação positiva, seguida por uma etapa em que a espécie autocatalítica B é removida, representando a retroalimentação negativa:

$$
\begin{gathered}
A+2 B \stackrel{k_{1}}{\longrightarrow} 3 B \\
B \stackrel{k_{2}}{\longrightarrow} C
\end{gathered}
$$

Aplicando os conceitos apresentados anteriormente, substituímos os valores da matriz $\mathrm{X}_{\mathrm{R}}$ correspondente (não mostrada) à Equação 9 e chegamos às leis de velocidade para cada etapa do mecanismo:

$$
\begin{aligned}
& v_{1}=k_{1}[A]^{1}[B]^{2}[C]^{0} \\
& v_{2}=k_{2}[A]^{0}[B]^{1}[C]^{0}
\end{aligned}
$$

As variações nas concentrações de A, B e C são obtidas substituindo-se os valores da matriz X correspondente na Equação 10 e adicionando-se a cada equação diferencial um termo para que leve em conta a entrada contínua dos reagentes A e B e a saída contínua tanto dos reagentes não consumidos, resultando no sistema de ODE'S:

$$
\begin{aligned}
& \frac{d[A]}{d t}=(-1) v_{1}+(0) v_{2}+k_{0}\left([A]_{0}-[A]\right) \\
& \frac{d[B]}{d t}=(1) v_{1}+(-1) v_{2}+k_{0}\left([B]_{0}-[B]\right) \\
& \frac{d[C]}{d t}=(0) v_{1}+(+1) v_{2}+k_{0}\left([C]_{0}-[C]\right)
\end{aligned}
$$

onde $[\mathrm{A}]_{0},[\mathrm{~B}]_{0}$, e $[\mathrm{C}]_{0}$ representam concentrações iniciais de A, B, e, $\mathrm{C}$, no reator, após mistura das soluções, mas antes que qualquer reação aconteça. Em outras palavras, as concentrações iniciais são os valores das concentrações das soluções que alimentam o reator divididos pelo número de canais da bomba peristáltica, considerando vazões iguais para cada canal. ${ }^{9}$

Os resultados da integração numérica do sistema de EDO's representado pelas Equações 20-22, utilizando-se dois valores para a razão de fluxo, são apresentados nas Figuras 4 e 5. Podemos verificar que a condução da reação em um sistema em fluxo muda completamente o comportamento da reação. Para razão fluxo $\mathrm{k}_{0}=0 \mathrm{~s}^{-1}$ (Figura 4), consistente com a simulação de uma reação em um reator em batelada, o sistema comporta-se como uma reação-relógio, com um curto período de indução, seguido de um salto na concentração de B, seguido por um decréscimo exponencial na concentração deste componente. Sob condições de fluxo (Figura 5), a mesma reação apresenta oscilações e biestabilidade, bem descritas na literatura. ${ }^{23}$

\section{Sistemas químicos oscilantes}

Os sistemas químicos oscilantes (SQO's) consistem em reações em que as concentrações dos intermediários variam periodicamente, tanto com o tempo, quanto com a posição..$^{9,10,22,23}$ Portanto, as concentrações de alguns componentes aumentam com o tempo até atingirem um valor máximo, quando diminuem a um valor mínimo, para então aumentarem novamente, oscilando ao longo do tempo. Dependendo da cinética e, também, das condições experimentais, os SQO's tanto podem apresentar oscilações periódicas bem comportadas, onde a mesma forma de onda se repete ao longo do tempo, quanto oscilações mais complexas. ${ }^{9,10,24}$ 


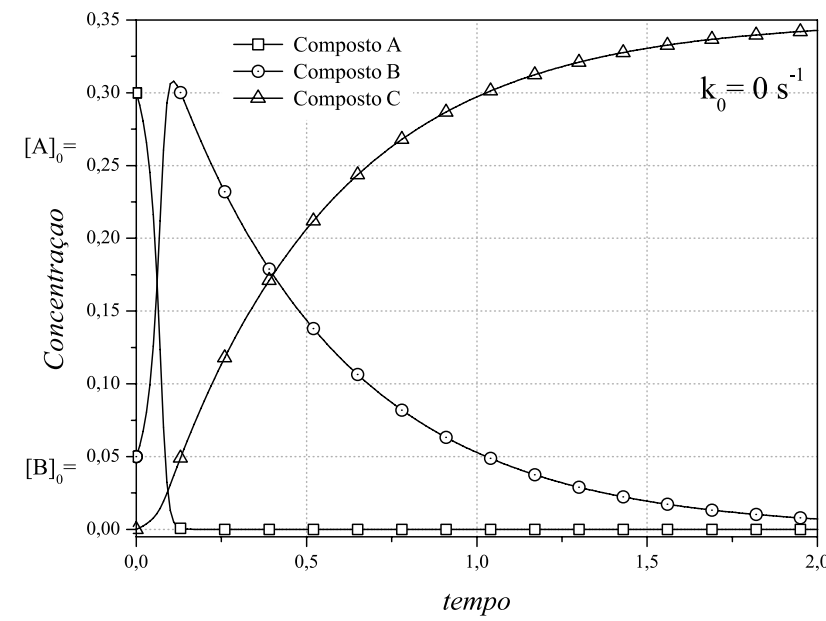

Figura 4. Perfis de concentração pelo tempo para uma reação constituída de uma etapa de autocatálise cúbica representando um processo de retroalimentação positiva, seguida por uma etapa em que a espécie autocatalítica $B$ é removida. Processo realizado em batelada (Razão de fluxo: $k_{0}=0 \mathrm{~s}^{-1}$ ) $[\mathrm{A}]_{0}$ $\left.=0,3 \mathrm{~mol} \mathrm{~L}{ }^{-1} ; B\right]_{0}=0,05 \mathrm{~mol} \mathrm{~L}{ }^{-1} ;[C]_{0}=0 \mathrm{~mol} \mathrm{~L} L^{-1} k_{1}=500 \cdot k_{2}$

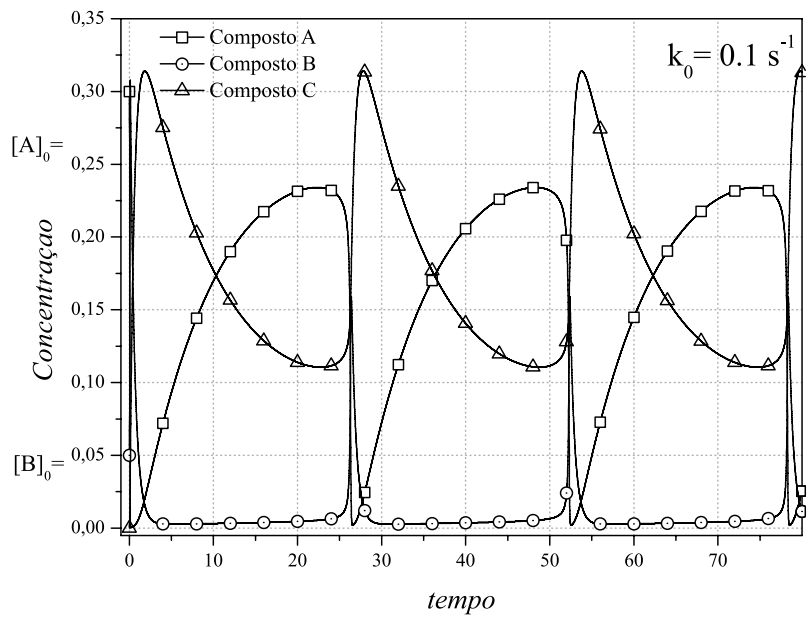

Figura 5. Perfis de concentração pelo tempo para uma reação constituída de uma etapa de autocatálise cúbica representando um processo de retroalimentação positiva, seguida por uma etapa em que a espécie autocatalítica $B$ é removida. Processo realizado em fluxo contínuo (Razão de fluxo: $k_{0}=$ $\left.0,1 \mathrm{~s}^{-1}\right):[A]_{0}=0,3 \mathrm{~mol} \mathrm{~L}{ }^{-1} ;[\mathrm{B}]_{0}=0,05 \mathrm{~mol} \mathrm{~L}{ }^{-1} ;[C]_{0}=0 \mathrm{~mol} \mathrm{~L} \mathrm{~L}^{-1} k_{1}=500 \cdot k_{2}$

Os SQO's possuem certas características em comum, como aparecimento das oscilações apenas em condições afastadas do equilíbrio; presença de etapas de retroalimentação positiva, na forma de uma reação autocatalítica, quando o produto de uma etapa elementar atua como catalisador na mesma etapa da reação; além de apresentarem o fenômeno de biestabilidade, que possibilita ao sistema assumir dois estados diferentes para o mesmo conjunto de condições iniciais, dependendo da história pregressa do sistema. Outro fator sempre presente nos SQO's é a não linearidade em suas leis de velocidade..$^{9,10,22-24}$

Tal importância no estudo e compreensão de SQO's está na variedade das situações onde estes sistemas se apresentam, que vão desde sistemas biológicos (ciclos hormonais, funções cardíacas) ${ }^{13,25}$ a reações de interesse comercial, como reações em reatores em batelada ${ }^{13,26}$ e reações eletroquímicas presentes em dispositivos conhecidos como célula a combustível. ${ }^{27}$ A maioria desses exemplos está longe de ser completamente compreendida, mas sistemas modelos podem trazer informações fundamentais que auxiliem compreender as diversas facetas dinâmico-matemáticas de sistemas complexos, bem como requisitos para interpretação química desses sistemas.

Para explorar as potencialidades do SCILAB ${ }^{19}$ na simulação dos perfis de concentração das espécies químicas envolvidas em SQO's, apresentaremos um exemplo envolvendo oscilações no $\mathrm{pH}$.

\section{Osciladores de $\mathrm{pH}$}

A família dos osciladores de $\mathrm{pH}$ consiste em SQO's nos quais são observadas grandes variações de $\mathrm{pH}$, ao mesmo tempo em que são a força motriz das reações, conduzidas em RFCA.22,28 Obviamente as oscilações são suprimidas em sistemas tamponados. Para haver oscilações de $\mathrm{pH}$, são necessários dois processos envolvendo os íons $\mathrm{H}^{+}$: produção autocatalítica de $\mathrm{H}^{+}$fazendo a vez da retroalimentação positiva e, após a devida defasagem temporal, consumo do $\mathrm{H}^{+}$, resultando em retroalimentação negativa. ${ }^{22,28}$

Soluções ácidas contendo íons bromato e sulfito, quando combinadas com uma espécie que consome próton, como o íon ferrocianeto, exibem oscilações de $\mathrm{pH}$ sob condições de fluxo. ${ }^{22,28}$ Para ilustrar o comportamento desse sistema, empregaremos um modelo proposto por Luo e Epstein ${ }^{28}$ que envolve quatro variáveis e cinco reações não elementares. Diferente de um mecanismo de reação, um modelo de reação é composto por processos não elementares e, eventualmente, não estequiométricos, no sentido que cada reação captura as características de várias etapas elementares do mecanismo real, identificando as etapas limitantes do mecanismo e assumindo que as demais etapas são arbitrariamente rápidas. ${ }^{10}$

Um elemento-chave do modelo é a produção autocatalítica de $\mathrm{H}^{+}$, representada pelas reações 6 e 7 :

$$
\begin{gathered}
\mathrm{A}+\mathrm{HX}+\mathrm{H} \rightarrow \mathrm{Y} \\
\mathrm{HX}+\mathrm{Y} \rightarrow 3 \mathrm{H}
\end{gathered}
$$

(reação 6) (reação 7)

onde A corresponde ao íon $\mathrm{BrO}_{3}^{-}, \mathrm{H}$ representa o íon $\mathrm{H}^{+}, \mathrm{X}$ representa $\mathrm{SO}_{3}^{2-}$ e Y, um ou mais intermediários, como $\mathrm{HBrO}_{2}, \mathrm{HOBr}$ ou $\mathrm{Br}_{2}{ }^{22,28}$

A retroalimentação negativa, responsável pela remoção do intermediário $\mathrm{Y}$ e íons $\mathrm{H}^{+}$, fica a cargo das reações:

$$
\begin{gathered}
\mathrm{Y} \rightarrow \mathrm{P} \\
\mathrm{B}+\mathrm{H} \rightarrow \mathrm{Q}
\end{gathered}
$$

(reação 8) (reação 9)

em que $\mathrm{P}$ representa o íon $\mathrm{Br}$ e $\mathrm{B}$ e $\mathrm{Q}$ representam os íons ferrocianeto e ferricianeto, respectivamente. ${ }^{22,28}$ Outro elemento-chave é o equilíbrio rápido de protonação, representado por

$$
H+X \rightleftharpoons H X
$$

(reação 10)

que reduz a concentração de $\mathrm{H}$, reduzindo assim a velocidade do processo autocatalítico e, por consequência, produzindo a defasagem temporal necessária entre as etapas de retroalimentação positiva e negativa. ${ }^{28}$ Os resultados da simulação são apresentados na Figura 6, mostrando as oscilações periódicas de $\mathrm{pH}$ e a concordância entre nossa simulação e os cálculos apresentados no artigo original. ${ }^{28}$ Vale ressaltar que na Figura 6 está representado apenas o logaritmo negativo da concentração de $\mathrm{H}^{+}\left(-\log H^{+}\right)$, os perfis de concentração para os 7 componentes restantes $(X, H X, P, B, Q e A)$ foram suprimidos de modo a deixar o gráfico mais compreensível. Outros osciladores de $\mathrm{pH}$ mais interessantes surgem, por exemplo, do sistema contendo $\mathrm{H}_{2} \mathrm{O}_{2}, \mathrm{Na}_{2} \mathrm{SO}_{3}, \mathrm{Na}_{2} \mathrm{CO}_{3}$ e $\mathrm{H}_{2} \mathrm{SO}_{4}$ em RAFC, onde é possível simular uma sequência de comportamentos complexos como a transição de um regime de oscilações periódicas a oscilações caóticas, passando por cascatas de dobra de período, a depender da razão de fluxo, ${ }^{29}$ mas este tema será abordado em trabalho futuro. 


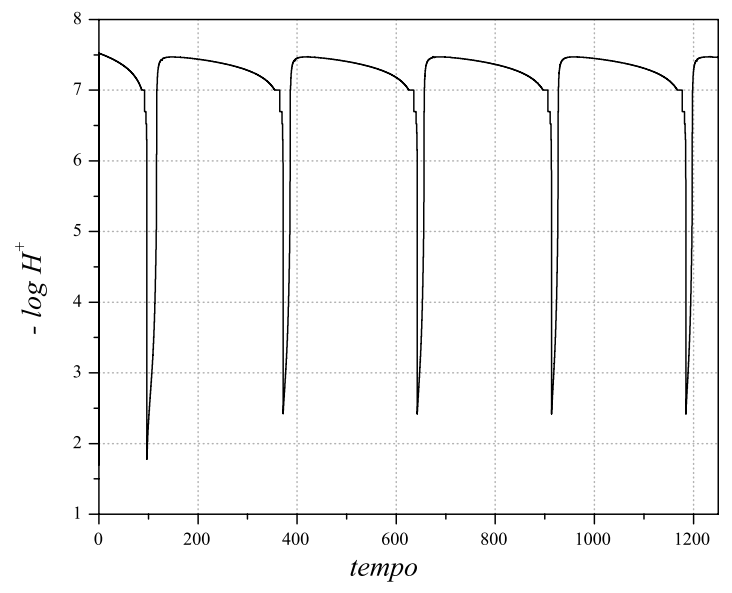

Figura 6. Variação do pH pelo tempo para o mecanismo de reação encontrado no oscilador bromato-sulfito-ferrocianato descrito pelas reações 6 a 10; $[\mathrm{H}]_{0}$ $=0,02 \mathrm{~mol} \mathrm{~L}^{-1} ;[X]_{0}=0,06 \mathrm{~mol} \mathrm{~L}^{-1},[\mathrm{HX}]_{0}=0,0 \mathrm{~mol} \mathrm{~L} L^{-1} ;[\mathrm{A}]_{0}=0,065 \mathrm{~mol}$ $L^{-1} ;[B]_{0}=0,02 \mathrm{~mol} \mathrm{~L} L^{-1} ;[Y]_{0}=0,0 \mathrm{~mol} \mathrm{~L} L^{-1} ; k_{1}=5 \times 10^{10} \mathrm{~mol}^{-1} \times L \cdot \mathrm{s}^{-1} ; k_{-1}=$ $3 \times 10^{3} \mathrm{~s}^{-1} ; k_{2}=3.077 \times 10^{6} \mathrm{~mol}^{-2} \times L^{2} \cdot \mathrm{s}^{-1} ; k_{3}=1 \times 10^{6} \mathrm{~mol}^{-1} \times L \cdot \mathrm{s}^{-1} ; k_{4}=11 \mathrm{~s}^{-1}$; $k_{5}=2,5 \mathrm{~mol}^{-1} \times L \cdot \mathrm{s}^{-1}$

\section{Limitações do modelo de cálculo utilizado}

Empregamos sistemas de equações diferenciais de primeira ordem para descrever as variações temporais nas concentrações das espécies químicas nos sistemas estudados. Nossas simulações são válidas estritamente para sistemas homogêneos isotérmicos, isto é, onde a agitação é eficiente para evitar gradientes espaciais e a temperatura é mantida constante. Para simular sistemas em que a temperatura é uma variável, seria necessário o emprego de equações transcendentais que levem em conta a dependência das constantes de velocidade com a temperatura, ao passo que para a simulação de sistemas não agitados, onde a presença de gradientes de concentração é certa, seria necessário o emprego de equações diferenciais parciais ao invés das equações diferenciais ordinárias que empregamos.

\section{CONCLUSÕES}

O uso de ferramentas computacionais é de extrema importância quando se trata de mecanismos reacionais compostos por inúmeras etapas, ou ordens superiores: as comumente apresentadas nos livros tradicionalmente utilizados no ensino de cinética química. Para isso utilizamos o SCILAB ${ }^{19}$ para escrever um software que auxiliasse aqueles que têm pouca familiaridade com métodos computacionais. Demonstramos sua aplicação em diversos exemplos de mecanismos reacionais, desde os mais simples até aos mais complexos como reações oscilantes, gerando gráficos dos perfis temporais de cada componente do mecanismo ou modelo reacional. A simulação cinética a partir do uso do software apresentado pode prover significantes discernimentos a respeito dos mecanismos de reações, que certamente se mostram um auxílio no ensino e no aprendizado desse tema.

\section{MATERIAL SUPLEMENTAR}

Para o auxílio do uso do software produzido disponibilizamos um tutorial onde o leitor encontrará o link para o Scilab, bem como o código de programação necessário para simular os mecanismos reacionais. Todo o material está disponível gratuitamente em http:// quimicanova.sbq.org.br, em arquivo pdf com acesso livre.

\section{AGRADECIMENTOS}

À COPES/UFS pela bolsa de inclusão em iniciação científica PIIC oferecida a R. D. D. Sandes.

\section{REFERÊNCIAS}

1. Mahan, B. M.; Myers, R. J.; Química um curso universitario, $4^{\mathrm{a}}$ ed.; Edgar Blucher: São Paulo, 1995.

2. Atkins, P. W.; De Paula, J.; Physical chemistry, $9^{\text {th }}$ ed.; W. H. Freeman and Co.: New York, 2010.

3. Ball, D. W.; Físico-Química, $1^{\mathrm{a}}$ ed.; Thomson Pioneira: São Paulo, 2005, vol. 1.

4. Moore, W. J.; Físico-Química, 4ª ed.; Blucher: São Paulo, 1976.

5. Monk, P. M. S.; Physical chemistry : understanding our chemical world, Wiley: Chichester, Hoboken, 2004.

6. Castellan, G. W.; Physical chemistry, $3^{\text {rd }}$ ed.; Addison Wesley: Reading, 1983.

7. Laidler, K. J.; Chemical kinetics, $3^{\text {rd }}$ ed.; Harper \& Row: New York, 1987.

8. Levine, I. N.; Fisicoquímica, 5 ed.; McGraw-Hill: Madrid, 2004.

9. Epstein, I. R.; Pojman, J. A.; An introduction to nonlinear chemical dynamics : oscillations, waves, patterns, and chaos, Oxford University Press: New York, 1998.

10. Scott, S. K.; Oscillations, waves, and chaos in chemical kinetics, Oxford University Press: Oxford, 1994.

11. Mortimer, R. G.: Mathematics for physical chemistry, $3^{\text {rd }}$ ed.; Elsevier Academic Press: Amsterdam, Oxford, 2005.

12. Puxty, G.; Maeder, M.; Hungerbuhler, K.; Chemom. Intell. Lab. Syst. 2006, $81,149$.

13. Elnashaie, S. S. E. H.; Uhlig, F.; Affane, C.; Numerical techniques for chemical and biological engineers using MATLAB: a simple bifurcation approach, Springer: New York, 2007.

14. Dyson, R.; Maeder, M.; Puxty, G.; Neuhold, Y. M.; Inorg. React. Mech. 2003, $5,39$.

15. Chodroff, L.; O’Neal, T. M.; Long, D. A.; Hemkin, S.; J. Chem. Educ. 2009, 86, 1072

16. Pfennig, B. W.; Roberts, R. T.; J. Chem. Educ. 2006, 83, 1804.

17. Francl, M. M.; J. Chem. Educ. 2004, 81, 1535.

18. Ferreira, M. M. C.; Ferreira Jr, W. C.; Lino, A. C. S.; Porto, M. E. G.; J. Chem. Educ. 1999, 76, 861.

19. Enterprises, S.; 5.XX ed. Orsay, France (www.scilab.org), 2012.

20. Barroso, L. C. B.; Magali M. A.; Campos, F. F.; Carvalho, M. L. B.; Maia, M. L.; Cálculo Numérico, 2a ed.; Editora Arbra: São Paulo, 1987.

21. Newberge, M.; Kadlec, R. H.; AIChE J. 1973, 19, 1272.

22. Sagues, F.; Epstein, I. R.; Dalton Trans. 2003, 1201.

23. Faria, R. de B.; Quim. Nova 1995, 18, 281.

24. Hall, N.; The new chemistry, Cambridge University Press: Cambridge, 2000.

25. Goldbeter, A.; Biochemical oscillations and cellular rhythms : the molecular bases of periodic and chaotic behaviour, Cambridge University Press: Cambridge, 1996.

26. Nogueira, P. A.; Varela, H.; Faria, R. B.; Chem. Phys. Lett. 2012, 530, 137.

27. Angelucci, C. A.; Varela, H.; Herrero, E.; Feliu, J. M.; J. Phys. Chem. C 2009, 113, 18835.

28. Luo, Y.; Epstein, I. R.; J. Am. Chem. Soc. 1991, 113, 1518.

29. Frerichs, G. A.; Thompson, R. C.; J. Phys. Chem. A 1998, 102, 8142. 


\section{INTEGRAÇÃO NUMÉRICA DE LEIS DE VELOCIDADE DIFERENCIAIS COM O USO DO SCILAB}

Rafael Donizete Dutra Sandes e Renato Canha Ambrosio*

Departamento de Química, Universidade Federal de Sergipe, Cidade Universitária Prof. José Aloísio de Campos, Av. Mal. Rondon, s/n, 49100-000 São Cristóvão - SE, Brasil

Camilo Andrea Angelucci

Centro de Ciências Naturais e Humanas, Universidade Federal do ABC, Rua Santa Adélia, 166, 09210-170 Santo André - SP, Brasil

\section{TUTORIAL PARA EXECUÇÃO DO SOFTWARE SKIN}

Para rodar o SKin o usuário necessitará de: Programa Scilab e Arquivos: Skin.sce e Skineq.sce.

$\mathrm{O}$ arquivo Skin.sce contém toda interface gráfica relacionada à digitação das matrizes $X_{R}$ e $X_{p}$, constantes, concentrações e intervalo de tempo. O arquivo Skineq.sce é utilizado durante a execução do Skin. sce para a criação do sistema de equações diferenciais. Atualmente o sistema funciona para mecanismos (ou modelos) de reação com até 11 etapas elementares e 11 espécies químicas. O SCILAB não gera um executável, mas interpreta as instruções contidas nos scripts Skin. sce e Skineq.sce que constituem o software que criamos.

\section{Primeiros passos}

1 - Primeiramente o usuário necessitará do programa SCILAB. Para isso entre no site http://www.scilab.org/products/scilab/download e faça o download para a plataforma utilizada. No nosso caso utilizamos o Windows 32-bits. Utilizamos o Scilab 5.3.3.

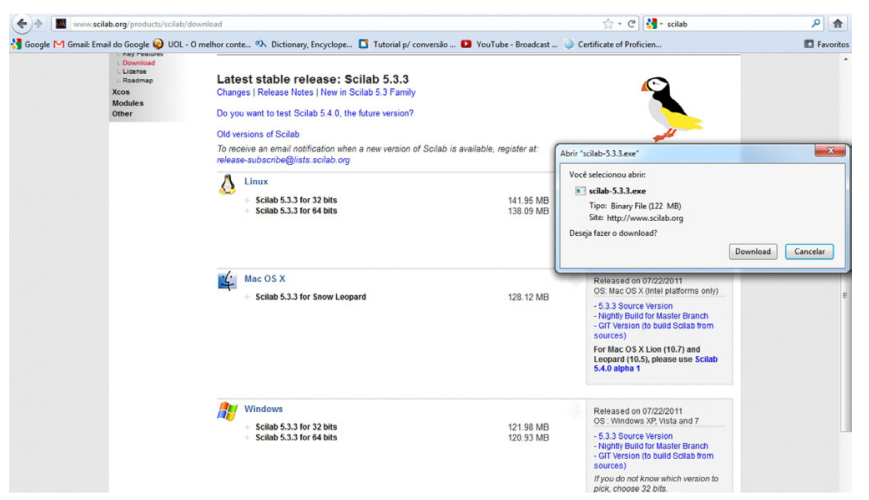

2 - Instale o SCILAB na pasta desejada e, em seguida, execute-o. A primeira tela a aparecer deverá ser como a seguinte:

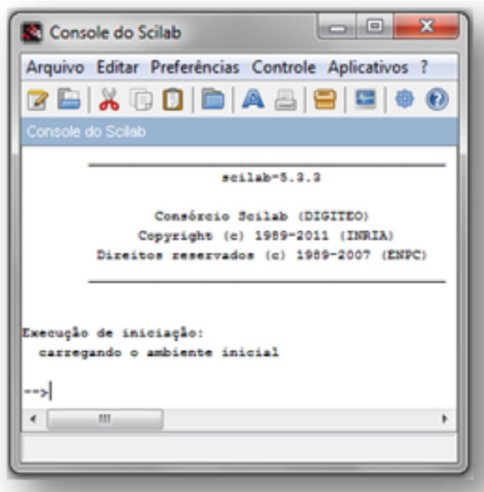

*e-mail: renatocanha@gmail.com
3 - Para utilizar o software Skin, é necessário instalar o pacote "Guimaker" que não vem instalado no SCILAB. Desta forma clique no ícone "Gerenciador de Módulos - Atoms.

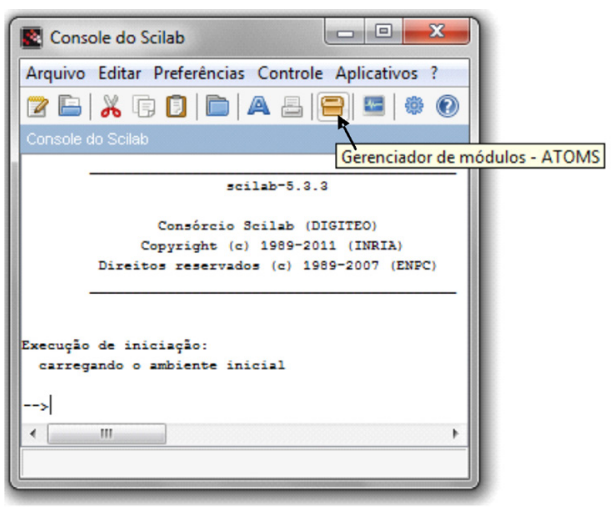

4 - Espere alguns segundos, pois o programa irá se conectar à internet. Serão abertas janelas, como mostrado abaixo. Em seguida clique em "GUI" e, depois, em "GUIMAKER".

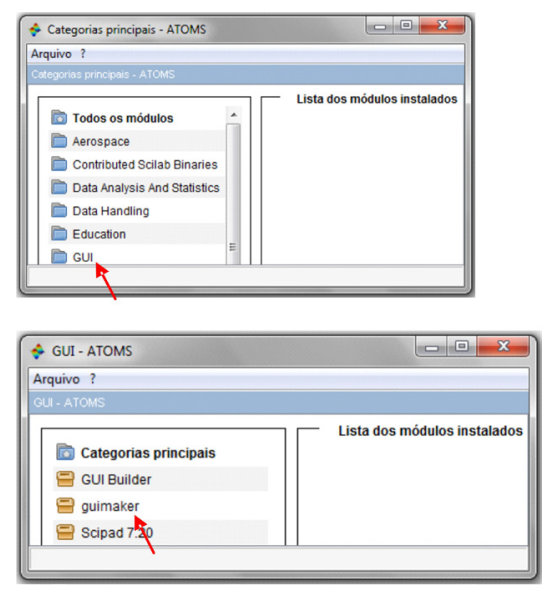

5 - Clique em Instalar (1) e assim que for concluído o processo o ícone "GUIMAKER" (2) que antes estava marrom ficará verde e aparecerá a mensagem "Instalação concluída! (3) Por favor, reinicie o Scilab para que as alterações tenham efeito".

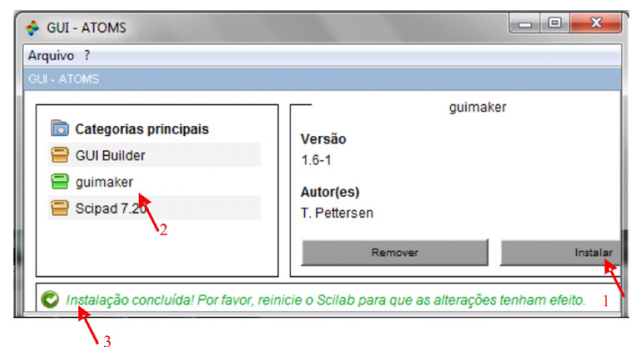


6 - Reinicie o programa para que possa iniciar as simulações.

\section{Abrindo o SKin}

1 - Crie uma pasta para que todos os dados gerados da simulação sejam salvos aí. No nosso caso criamos a pasta "Códigos de Programa", mas fica a critério do usuário escolher o nome e a localização da pasta. Coloque os arquivos Skin.sce e Skineq.sce na pasta criada. Aqueles que desejarem, poderão simplesmente copiar os códigos que são fornecidos no final deste documento para o scinotes e salvar no diretório recém-criado.

2 - Como indicado abaixo, altere o diretório atual do Scilab para a pasta criada no ítem 1 .
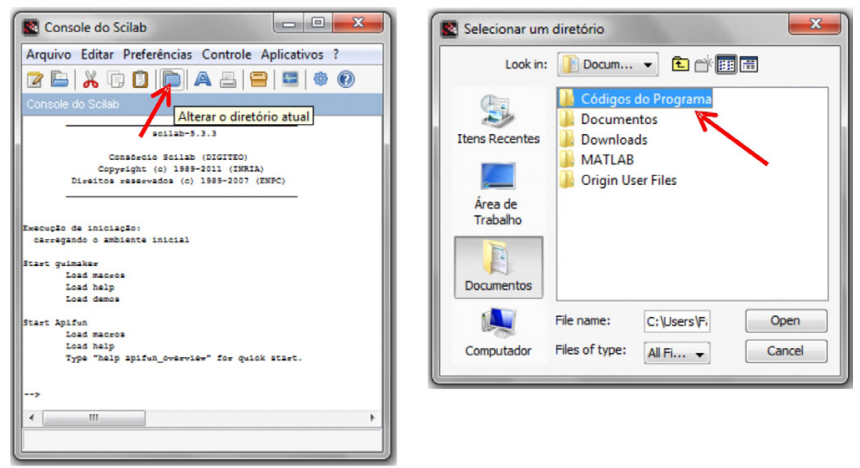

3 -Agora, no Console do Scilab no menu superior esquerdo clique em "Arquivo"/ "Abrir um Arquivo" (ou digite CTRL+O)

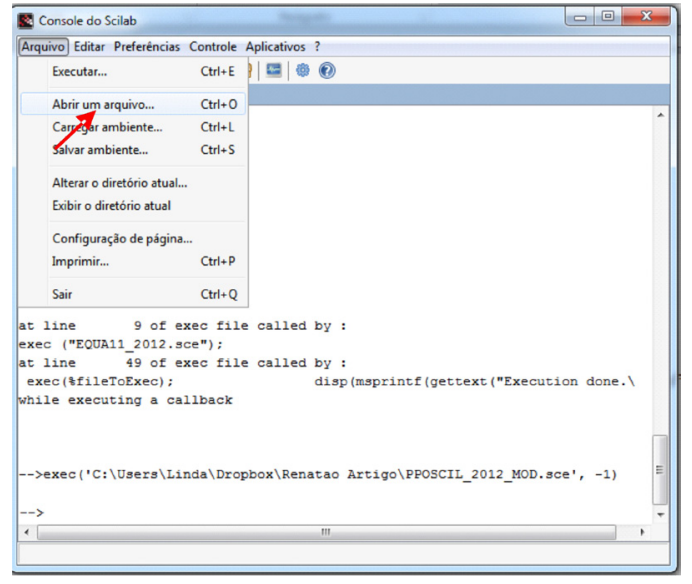

4 - Selecione o arquivo Skin.sce e clique em abrir. A seguinte janela será aberta:

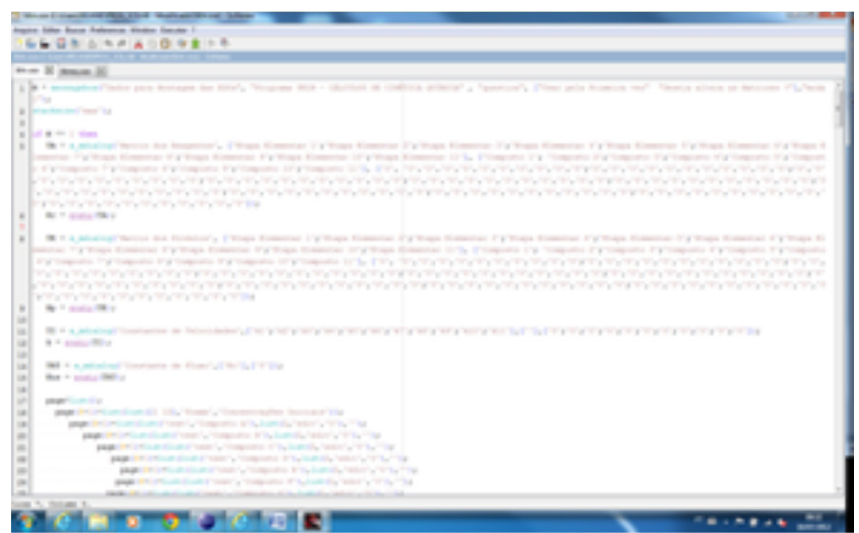

Você poderá visualizar o código de programação em SCILAB. Caso queira visualizar o código responsável pela montagem das equações diferenciais, basta abrir o arquivo Skineq.sce.

\section{Simulando}

Para nos ambientalizarmos ao SKin, vamos começar com um mecanismo de reações consecutivas, competitivas de segunda ordem, representados pelas reações 2 e 3 apresentadas no texto do artigo.

Para iniciar a simulação é necessário montar as equações diferenciais que regem o mecanismo. Devemos lembrar que o próprio SKin faz isso para você, mas para tanto é necessário que o usuário introduza os dados corretos.

1 - De volta a janela do scinotes (que já está aberto com o arquivo Skin.sce clique em "Executar" ( ou aperte F5).

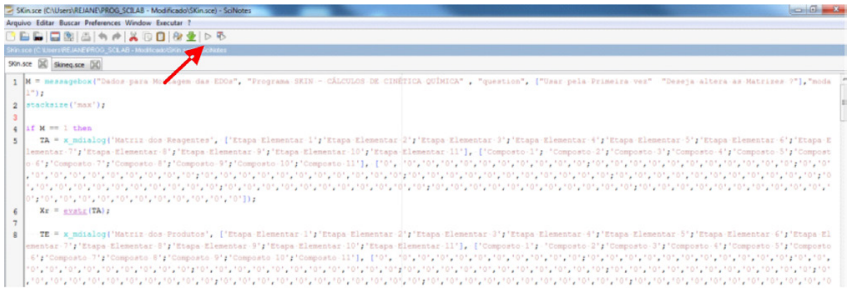

2 - O programa exibirá a seguinte caixa de diálogo:

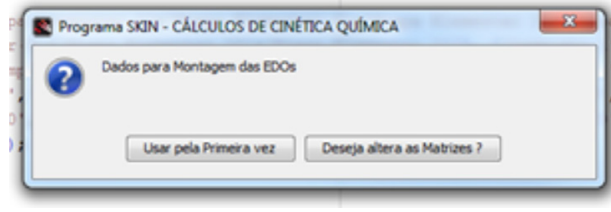

Clique no primeiro botão. O segundo será necessário caso o usuário deseje refazer os cálculos modificando, por exemplo, concentrações iniciais, valores das constantes ou qualquer outro parâmetro relativo ao mecanismo simulado.

3 - Abrirá uma janela para ser construída a matriz $X_{R}$. Nesta janela o usuário deverá introduzir os coeficientes estequiométricos dos reagentes de cada etapa elementar presente no mecanismo. O Skin é capaz de trabalhar com um mecanismo reacional de até 11 etapas elementares contendo 11 compostos. Por padrão todos os componentes de todas as etapas já vêm como zero (ou nulo). Digite os valores correspondentes da matrix $\mathrm{Xr}$ :

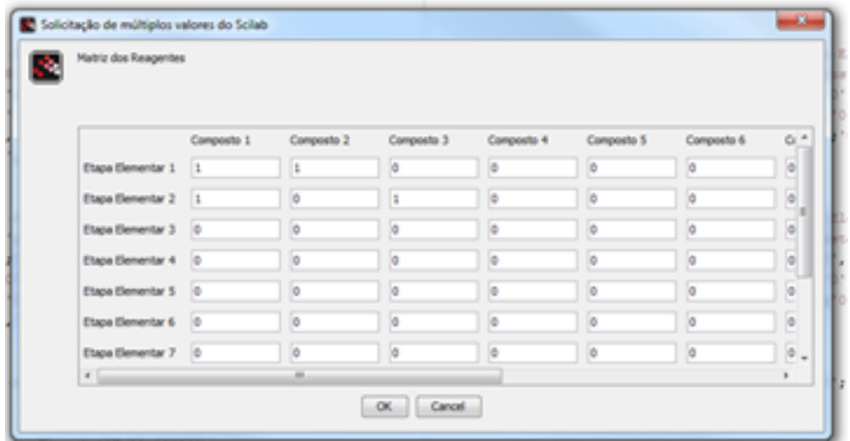

4 - Clique em OK e então abrirá a janela da matriz $\mathrm{X}_{\mathrm{P}}$. Após ser preenchida resulta em: 


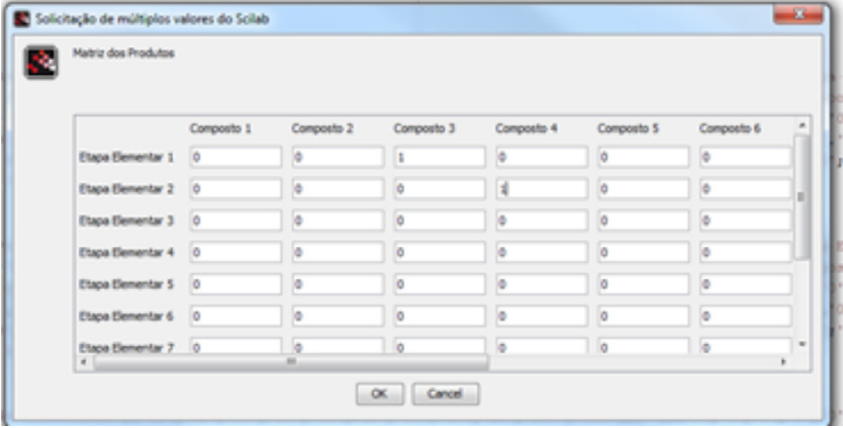

5- A janela de constantes de velocidade será aberta e o usuário introduzirá os valores das constantes de velocidade para cada etapa elementar. Após digitar, clique em OK.

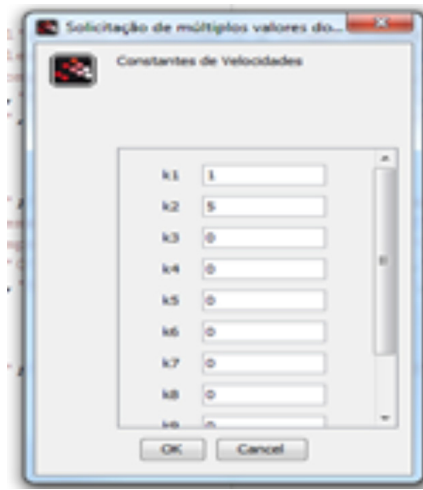

6-Em seguida abrirá a janela para constante de fluxo. Como neste exemplo não há entrada nem saída de componentes (sistema fechado, ao contrário do RFCA) deixe com o valor zero e clique em OK.

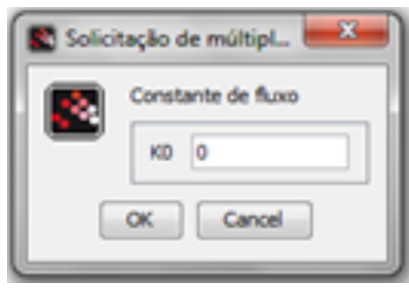

7 - Uma nova janela será aberta para a digitação das concentrações iniciais de cada componente. A abertura dessa janela pode levar de 10 a $20 \mathrm{~s}$. Para o nosso exemplo utilizamos $1 \mathrm{~mol} \mathrm{~L}^{-1}$ para o componente A e $0,5 \mathrm{~mol} \mathrm{~L}^{-1}$ para o componente $\mathrm{B}$ (digite 0.5 pois o programa não reconhece vírgula).

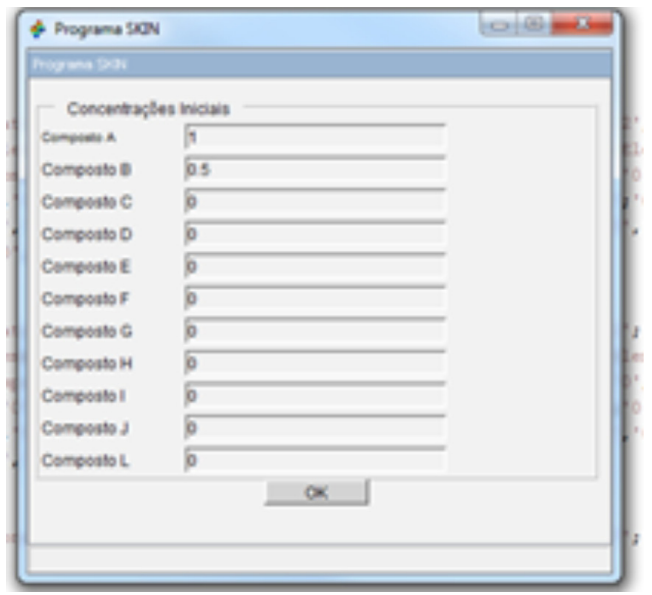

8 - Clique em OK.

9 - Serão solicitados os tempos inciais e finais para o processo. Digite 10 no xmax, E clique OK.

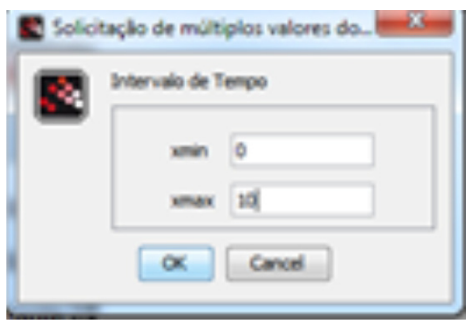

10 - Clique em OK na janela seguinte para iniciar o processamento. Dependendo do número de equações, pode demorar de alguns segundos a minutos.

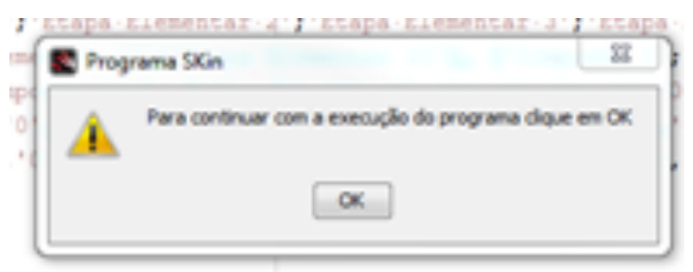

11-Aparecerá a janela perguntando sobre a parte gráfica. O SKin proporciona ao usuário visualizar os dados gerados. Clique em Sim.

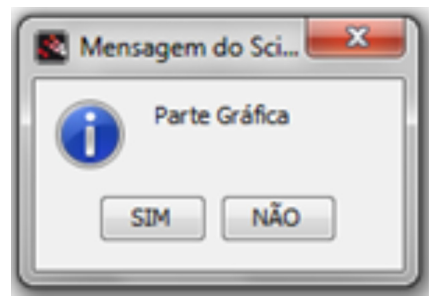

12- Será apresentado o perfil temporal de cada componente presente no mecanismo reacional. Clique no gráfico para que as legendas apareçam. O usuário pode escolher visualizar cada componente separadamente selecionando-o na janela anexa à janela gráfica.

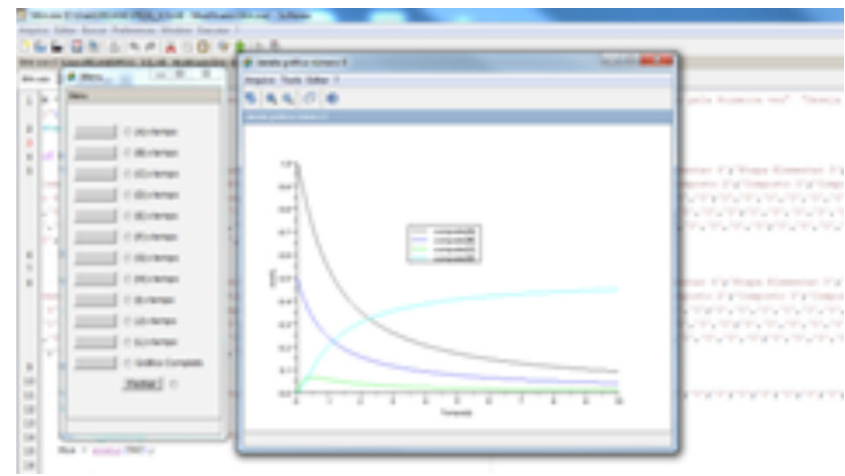

Após observar os perfis de concentração de cada componente, é importante fechar a janéla de opções gráficas para que o programa exiba a mensagem de salvar os dados.

13- O Skin salvará os dados gerados se o usuário apertar a opção Sim 


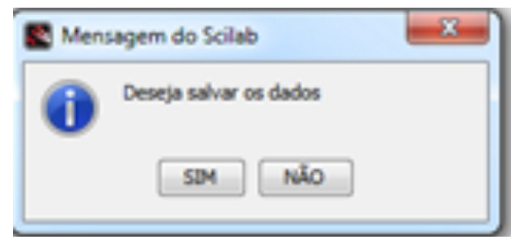

Os dados podem ser salvos nos formatos xls, dat e txt. Após salvar os dados, o programa permite que o usuário refaça os cálculos, sem a necessidade de digitar as matrizes novamente, sendo necessário apenas digitar os dados que deseja alterar.

14- $\mathrm{O}$ local e o nome do arquivo fica a critério do usuário. $\mathrm{O}$ arquivo final pode ser aberto por qualquer programa comercial de tratamento de dados, como Origin, Excel, Quiplot, etc, sendo a primeira coluna o tempo e as demais colunas o composto A, B e assim por diante.

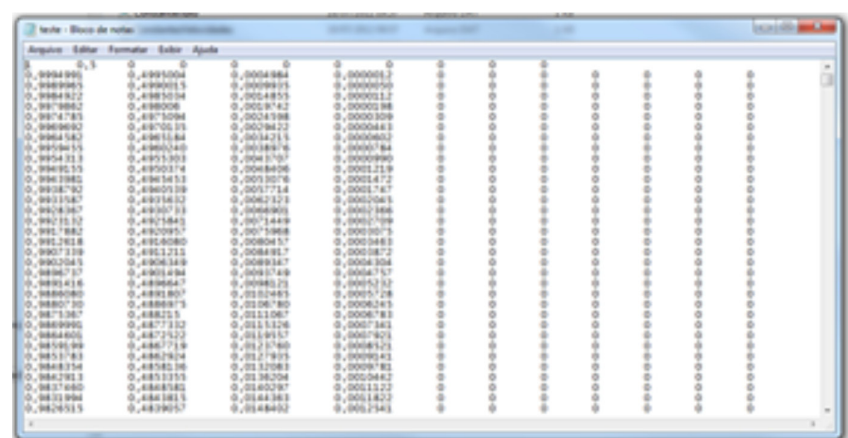

Nota: Como o código é aberto, qualquer usuário pode modificá-lo, embora os autores não o recomendem. Recomendamos o uso do código apenas para fins educacionais e demonstrativos. Não oferecemos nenhum tipo de garantia quanto a usos diferentes dos propostos neste documento.
Exemplo para os usuários praticarem. oscilador de $\mathrm{pH}$

$$
\begin{aligned}
& \text { (01) } \mathrm{SO}_{3}^{2}+\mathrm{H}^{*} \rightarrow \mathrm{HSO} ; \quad k_{1}-5 \times 10^{10} \mathrm{mor}^{-1} \mathrm{Ls}^{-1} \\
& \text { (02) } \quad \mathrm{HSO}_{3} \rightarrow \mathrm{SO}_{3}^{*}+\mathrm{H}^{-} \quad k_{-1}-3 \times 10^{\circ} \mathrm{s}^{-1} \\
& \text { (03) } \quad \mathrm{BrO}_{3}+\mathrm{HSO}_{1}+\mathrm{H}^{*} \rightarrow \mathrm{Y} \quad k_{2}-3.077 \times 10^{\circ} \mathrm{mo}^{-2} \mathrm{Ls}^{-2} \\
& \text { (04) } \mathrm{HSO}_{2}+\mathrm{Y} \rightarrow 3 \mathrm{H}^{-} \quad k,-1 \times 10^{\circ} \\
& \text { (05) } \quad \mathrm{Y} \rightarrow \mathrm{P} \quad k_{i}-115^{-1} \\
& \text { (05) } \mathrm{B}+\mathrm{H} \rightarrow \mathrm{Q} \quad k_{2}-2.5 \mathrm{~mol}^{-1} L \mathrm{~s}^{\mathrm{a}}
\end{aligned}
$$

\begin{tabular}{|c|c|c|c|c|c|c|c|c|}
\hline & $\mathrm{SO}^{2}$ & $\mathrm{H}^{-}$ & $\mathrm{HSO}$ & $\mathrm{BrO}$; & Y & P & $\bar{B}$ & $\bar{Q}$ \\
\hline 1.Eapa Ėemectar & 1 & $\overline{1}$ & 0 & $\overline{0}$ & $\overline{0}$ & 0 & $\overline{0}$ & 0 \\
\hline $2^{*}$ Etapa Elemectar & 0 & 0 & 1 & 0 & 0 & 0 & 0 & 0 \\
\hline $3^{*}$ Eapa Elemectar & 0 & i & 1 & i & 0 & 0 & 0 & 0 \\
\hline 4" Esopa Eismectar & 0 & 0 & 1 & 0 & 1 & 0 & 0 & 0 \\
\hline 5* Espa Eismeetar & 0 & 0 & 0 & 0 & 1 & 0 & 0 & 0 \\
\hline $6^{*}$ Etapa Elemectar & 0 & 1 & 0 & 0 & 0 & 0 & 1 & 0 \\
\hline
\end{tabular}

\begin{tabular}{|c|c|c|c|c|c|c|c|c|}
\hline & $\mathrm{SO}^{2}$ & $\mathrm{H}^{*}$ & $\mathrm{HSO}_{\text {; }}$ & $\mathrm{BrO}$; & $Y$ & p & $\bar{B}$ & $Q$ \\
\hline 1"Espa Elemectar & 0 & $\overline{0}$ & 1 & 0 & $\overline{0}$ & $\overline{0}$ & 0 & 0 \\
\hline 2* Etapa Elemectar & 1 & 1 & 0 & 0 & 0 & 0 & 0 & 0 \\
\hline 3* Etapa Elemectar & 0 & 0 & 0 & 0 & 1 & 0 & 0 & 0 \\
\hline 4* Etaps Elemectar & 0 & 3 & 0 & 0 & 0 & 0 & 0 & 0 \\
\hline 5* Espo Eiemecas & 0 & 0 & 0 & 0 & 0 & 1 & 0 & 0 \\
\hline $6^{*}$ Enapa Elemeetar & 0 & 0 & 0 & 0 & 0 & 0 & 0 & 1 \\
\hline
\end{tabular}

$k_{0}-10^{2}$ Cocsacts de faxo;

\begin{tabular}{cc}
\hline Especies & Concentraçóes Iniciais(MI) \\
\hline $\mathrm{SO}^{2}$ & 0.06 \\
$\mathrm{H}^{-}$ & 0.02 \\
$\mathrm{HSO}^{-}$ & 0 \\
$\mathrm{BrO}^{\prime}$ & 0.065 \\
$\mathrm{Y}$ & 0 \\
$\mathrm{p}$ & 0 \\
$\mathrm{~B}$ & 0.02 \\
$\mathrm{Q}$ & 0 \\
\hline
\end{tabular}

TABELA DOS REAGENTES:

TABEL A DOS PRODUTOS: 